\title{
Author Index (Volume 25)
}

Abbes, M. 1750035

Abboudi, S. 1750022

Ahn, S. W. 1750008

Akisawa, A. 1750028

Albouchi, A. 1750001

Ameri, M. 1750016

Anas, N. S. 1750017

Aridhi, E. 1750001,1750035

Banker, N. D. 1750032

Biju, T. K. 1750031

Boahen, S. 1750024

Byun, K. H. 1750034

Chang, K. S. 1750006

Chao, C. 1750020

Cho, H. 1730001, 1750013

Cho, S. 1750024

Choi, J. M. 1750010, 1750024

Choi, K.-I. 1750013

Chung, J. D. 1750011

Clodic, D. 1750009

Dandotiya, D. 1750032

Dasgupta, M. S. 1750012

Descombes, G. 1750009

Doiphode, P. 1750026

Duong, X. Q. 1750011

Enoki, K. 1750028

Gill, J. 1750015

Govinde Gowda, M. S. 1750023
Gowda, M. S. G. 1750002

Guo, Z. 1750020

Han, H. 1750021

Heo, J. 1750030

Hu, J. 1750020

Jani, D. B. 1730002

Jeong, J. H. 1730004

Kang, B. H. 1730003

Kang, D. H. 1730005

Kan, A. 1750020

Kariya, K. 1750004, 1750027

Keryakos, E. 1750009

Khaldi, S. 1750022

Kim, B. G. 1750018

Kim, D. 1750018

Kim, H. 1730001

Kim, J. 1730001

Kim, M. J. 1750006

Kim, M. S. 1730005

Kim, M.-H. 1750030

Kim, N.-H. 1750025

Kim, Y. J. 1750006

Kim, Y. 1750018

Kishor Kumar, V. V. 1750014

Korti, A. I. N. 1750022, 1750033

Korti, A. I. N. 1750033

Kumar, M. 1750026

Kuzhiveli, B. T. 1750014

Kwon, Y.-I. 1750029
Lee, D. 1750018

Lee, D.-W. 1750030

Lee, H. J. 1730003

Lee, K. H. 1750024

Lee, M. S. 1750008

Mahmud, M. S. 1750004

Mami, A. 1750035,1750001

Matsumoto, M. 1750003

Meng, C. 1750020

Mensah, K. 1750010

Mishra, M. 1730002

Miyara, A. 1750004,1750027

Mohammadi, S. M. H. 1750016

Na, S.-I. 1730005

Narasimham, G. S. V. L. 1750002

Narasimham, G. S. V. L. 1750023

Ogawa, K. 1750003

Oh, J.-T. 1750013

Pendyala, S. 1750019

Peterson, G. P. 1750017

Prah, B. 1750005

Prashantha, B. G. 1750002, 1750023

Prattipati, R. 1750019

Rachedi, K. 1750033

Rahman, H. 1750021

Rahman, M. M. 1750027 
Rajesh, V. R. 1750031

Raju, A. V. S. R. 1750019

Rasti, M. 1730004

Sahoo, P. K. 1730002

Samanta, I. 1750026

Seetharamu, S. 1750023, 1750002

Senthilkumar, D. 1750007
Shoul, H. 1750016

Singh, J. 1750015

Singh, S. 1750012

Sobhan, C. B. 1750017

Takei, T. 1750028

Thomas, S. 1750017

Toubassy, J. 1750009
Vu, P.-Q. 1750013

Wakabayashi, H. 1750003

Watanabe, F. 1750028

Yasumoto, Y. 1750003

Yun, R. 1750030, 1750005 